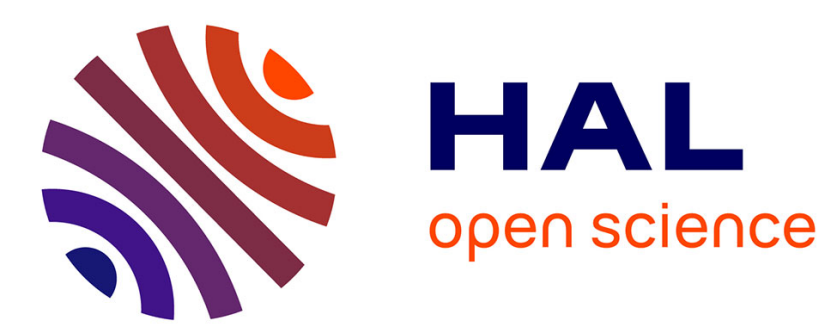

\title{
Evidence for water ridges at oil-water interfaces: implications for ion transport
}

Boyao Wen, Chengzhen Sun, Wenxiu Zheng, Bofeng Bai, Eric Lichtfouse

\section{To cite this version:}

Boyao Wen, Chengzhen Sun, Wenxiu Zheng, Bofeng Bai, Eric Lichtfouse. Evidence for water ridges at oil-water interfaces: implications for ion transport. Soft Matter, 2020, 16, pp.826 - 832. 10.1039/c9sm01791g . hal-03040104

\section{HAL Id: hal-03040104 https://hal.science/hal-03040104}

Submitted on 4 Dec 2020

HAL is a multi-disciplinary open access archive for the deposit and dissemination of scientific research documents, whether they are published or not. The documents may come from teaching and research institutions in France or abroad, or from public or private research centers.
L'archive ouverte pluridisciplinaire HAL, est destinée au dépôt et à la diffusion de documents scientifiques de niveau recherche, publiés ou non, émanant des établissements d'enseignement et de recherche français ou étrangers, des laboratoires publics ou privés. 


\title{
Evidence for water ridges at oil-water interfaces: implications for ion transport $\uparrow$
}

\author{
Boyao Wen, Chengzhen Sun, (D) Wenxiu Zheng, Bofeng Bai (D)* and \\ Eric Lichtfouse (D)
}

Understanding ion transport across interfaces is of fundamental importance in many processes such as liquid-liquid extraction, phase transfer catalysis, enhanced oil recovery and emulsion stabilisation. However, the factors that control ion transport across interfaces are poorly known due to a lack of knowledge of structural changes at interfaces. We studied here the effects of ionic concentration and external force on the transport of ions across the decane-water interface using classical molecular dynamics simulations. The results show that the evolution of interfacial structures during ion transfer across the interface is controlled by hydrogen bonding and ionic interactions at the interface. We also identified a new mode of ion transfer across the interface at low ionic concentrations, involving a 'water ridge', rather that the classical 'water finger'. In the water ridge mode, hydrogen bonds are not broken due to low ion levels, and the water ridge induces gradual interface deformation. Whereas, at high ionic concentrations, hydrogen bonds are broken by the strong ion electrostatic repulsion, thus inducing the formation of a water finger. We also found that the variation of the Gibbs free energy during ion transfer is directly relevant to the ionic concentration. The water ridge at low ionic concentrations, which displaces more water molecules towards the decane phase, induces less free energy variation than the water finger at high ionic concentrations.

\section{Introduction}

Ion transport across the interface between two immiscible liquids is a fundamental phenomenon which plays a major role in many industrial processes, such as electrochemistry, liquid-liquid extraction, phase transfer catalysis and enhanced oil recovery. ${ }^{1-5}$ Since the interface is a narrow region with properties markedly different from the bulk phase, the characteristics of ion transport at interfaces are different from those of the bulk phase. The transport of ions across interfaces is controlled by many factors such as the liquid components, concentration, interfacial adsorbing materials, and the external environment. ${ }^{6-10}$

Experimental and theoretical investigations have been recently launched to decipher the mechanisms of ion transport across the oil-water interface. Molecular-level insights have been revealed using advanced techniques such as nonlinear optical spectroscopy, ${ }^{11,12}$ potential-step chronofluorometry, ${ }^{13} \mathrm{X}$-ray diffraction and reflection ${ }^{14}$ and neutron reflection. ${ }^{15}$ The effect of temperature, ionic concentration and applied potential on the transport rates of ions across interfaces has been studied. The results have shown that ion transport is kinetically controlled at low temperature, and the

State Key Laboratory of Multiphase Flow in Power Engineering, Xi'an Jiaotong University, Xi'an, Shaanxi, 710049, China. E-mail: bfbai@mail.xjtu.edu.cn; Tel: +86-029-82665316

$\dagger$ Electronic supplementary information (ESI) available. See DOI: 10.1039/c9sm01791g
Gibbs energy of ion transfer is apparently unrelated to ion transport rates. ${ }^{16,17}$ Kakiuchi and Takasu further assumed that the ion transport rate is mainly controlled by the applied potential. ${ }^{18}$

Molecular dynamics (MD) simulations have been conducted due to the limitations of laboratory experiments to gain information on interfacial dynamics. MD simulations have thus disclosed information on ion solvation, interfacial microstructures and other molecular details. ${ }^{19-23}$ For instance, Wick and Xantheas ${ }^{24}$ found that the anisotropy of solvated structures at the interface is consistent with the polarizability and inversely correlated with the size of the anions. They also presented the factors that control ion behaviour at the interface, and discussed the effect of ion pairing on the interfacial behaviour and on free energies. ${ }^{25,26}$ Indeed, ion adsorption, desorption, distribution and transport are major processes controlling the interfacial structure, deformation and mechanical properties. ${ }^{27-31}$ In our previous work, we found that the adsorption of sodium chloride at the decane-water interface causes a non-monotonic increase of the interfacial tension. ${ }^{32} \mathrm{We}$ also demonstrated that the reduction of interfacial tension is sensitive to the cation species due to the ionic hydration at the interface. ${ }^{33}$ Combining MD simulation and mean field theory, Luo et al. ${ }^{34}$ obtained the distributions of ions near the interface, which agreed with experiments.

Ion transfer across oil-water interfaces involves the change of the ion solvation ability in different liquid phases, which is usually described by the variation of the solvation free energy. 
Hence, the free energy change during ion transfer across interfaces, which is comparable with experimental data, can give the energetic features and mechanisms of ion transfer. In the free energy calculation, the reaction coordinate plays a crucial role. The most common coordinate is the coordinate of the ion normal to the interface, namely $z$, where the Gibbs dividing surface is treated as the interface between the oil and the water phase..$^{35-41}$ Benjamin $^{35}$ initially used MD simulations to demonstrate that ion transfer across the interface is an activated process which is governed by a free energy barrier. Nonetheless, Fernandes et al. $^{42}$ proposed that ion transport does not require activation due the absence of a free energy barrier. They explained the mechanism of ion transport by the formation of a water finger, that is a chain of water molecules connecting the hydration shell of ions to the water bulk phase. Similarly, Dos Santos and Gomes ${ }^{38}$ assessed the free energy and the $\mathrm{Ca}^{2+}$ transport process during the formation of a water finger, and found that ion transport across the interface is not activated. Using the $z$ coordinate assumes that the ion moves in the mean field of two solvents and the interfacial fluctuations can be neglected. However, the transfer of ions across the interface is generally accompanied by interfacial fluctuations and deformation. Meanwhile, the distribution of ions at interfaces is usually uneven. Thereby, it is difficult to accurately calculate the free energy using only the $z$ coordinate. Recently, several additional coordinates which can consider the interface deformation and fluctuations were proposed. For example, an energy-based solvation coordinate presented by Schweighofer and Benjamin, ${ }^{43}$ a geometrical coordinate $\Lambda$ reported by Kornyshev et $a .^{44}$ and the water-finger coordinate $w$ raised by Kikkawa et al. ${ }^{45}$ Among them, Kikkawa et al. ${ }^{45-49}$ obtained the 2D free energy surface by using a water-finger coordinate $w$ to describe the formation and the break of a water finger, and revealed a hidden barrier of ion passage associated with the water finger. Two coordinate methods, which can consider more factors, such as ion hydration, ionic interactions, ion pairs, interfacial films, etc., need further investigation.

Generally, it is accepted that the mechanism of ion transport across the oil-water interface is directly related to the formation of a water finger. The formation of a water finger during ion transport across the interface is controlled by other factors such as the oil components, ${ }^{50}$ ion properties, ${ }^{51}$ ion pairing ${ }^{40}$ and fluctuation of the interface. ${ }^{52}$ The transport of ions across the interface is usually initiated by compounds such as surfactants and lipophilic complexes, inducing the formation of specific interfacial structures, for instance a water channel. ${ }^{51,53,54}$ These specific interfacial structures bring a challenge for the free energy calculation. Recently, combined with MD simulations, some enhanced sampling methods such as replica exchange, ${ }^{55}$ accelerated molecular dynamics, ${ }^{56}$ umbrella sampling, ${ }^{57}$ and metadynamics ${ }^{58}$ have been proposed and developed to obtain a more accurate description of the free energy during ion transfer across interfaces.

In summary, previous research has focused on free energy changes during the formation of a water finger, thus giving information on the transport resistance. However, the molecular details of ion transport and the effect of the ionic concentration and external forces are poorly known. Moreover, how the interfacial structures develop during ion transport across the interface is still debated. This information plays a significant role in the calculation of the free energy, which can further reveal the hidden mechanism of ion transfer across interfaces. Therefore, here we performed MD simulations of ion transport across the decanewater interface to gain information on the microscopic details and contributing factors.

\section{Model and methods}

\section{Simulation model}

We studied the transport processes of ions across the interfaces in a 'sandwich' MD simulation system, where the oil phase is modelled by decane according to the equivalent alkane carbon number of the crude oil. ${ }^{59}$ Fig. 1 shows the MD simulation system, composed of layers of brine (water and ions), water and decane phases. This system has two planar interfaces that are perpendicular to the $z$-direction. In order to neglect the interaction between interfaces, the thickness of the decane and water phases must be large enough, e.g. higher than $5 \mathrm{~nm}$. The volume of the initial simulation model was $350 \mathrm{~nm}^{3}\left(L_{x}\right.$ and $\left.L_{y} 5 \mathrm{~nm}, L_{z} 14 \mathrm{~nm}\right)$. As the basic components of brine and fluids in processes, $\mathrm{Na}^{+}$and $\mathrm{Cl}^{-}$ions are used and uniformly dissolved in the brine phase. For the sake of fast separation between oppositely charged ions and eliminating the effect of ion pairing on the ion transport across the interfaces, brine phases with thicknesses of $1 \mathrm{~nm}$ are arranged at both ends. Five ionic concentrations $c$ of $0.1,0.2,0.3,0.4$ and $0.5 \mathrm{~mol} \mathrm{~L}^{-1}$ are simulated to test the effect of ionic concentration on the transport of ions across the interface. 3D periodic boundary conditions are applied for all simulations.

\section{Force field parameters}

The force fields which describe the interactions between atoms play a dominant role in the movement of molecules and the statistical properties of the system. In our simulations, all atomic interactions between atoms involving van der Waals attractions, electrostatic interactions, bond stretching, angle bending and torsion interactions are considered. The non-bonded interaction energy $U$ between atoms $i$ and $j$, possessing partial charges $q_{i}$ and $q_{j}$ respectively, is described by the conventional

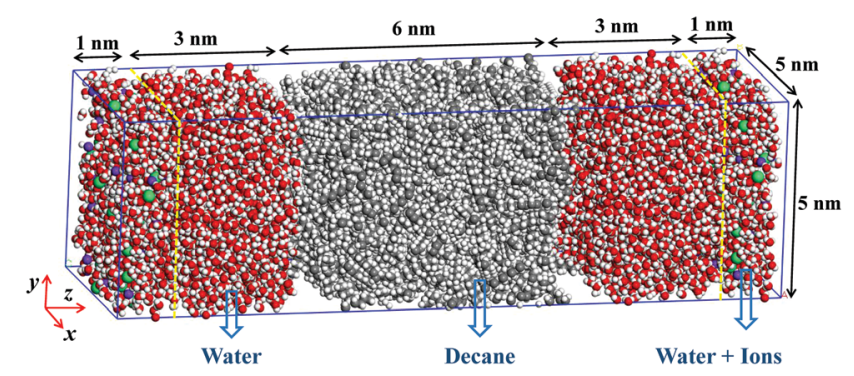

Fig. 1 Molecular dynamics (MD) simulation system. Red: oxygen atoms; white: hydrogen atoms; purple: sodium ions; green: chloride ions. 
12-6 Lennard-Jones (L-J) and Coulombic pairwise interactions, given by:

$$
U_{i j}=4 \varepsilon\left[\left(\frac{\sigma}{r_{i j}}\right)^{12}-\left(\frac{\sigma}{r_{i j}}\right)^{6}\right]+\frac{q_{i} q_{j} e^{2}}{4 \pi \varepsilon_{0} r_{i j}} \quad\left(r_{i j}<r_{\mathrm{cut}}\right)
$$

where $\varepsilon$ is the depth of the potential well, $\sigma$ is the zero interatom potential energy location, $r_{\text {cut }}$ is the cut-off distance, and $\varepsilon_{0}$ is the dielectric constant. The non-bonded interactions between water molecules are depicted by the TIP3P model ${ }^{60,61}$ while the OPLS-AA model ${ }^{62}$ is used to accurately reproduce the thermodynamic properties of the decane phase. In our previous work, ${ }^{30,31}$ the simulation results of these potentials prove their ability to accurately reproduce the thermodynamic and interfacial properties of water and decane, such as the density, diffusion coefficients and interfacial tension. The Lorentz-Berthelot rule is employed to get the L-J potential parameters between different atoms (ESI 1.1 $\dagger$ ).

We used a cut-off distance of $10 \AA$ for all non-bonded interactions in the simulations. Considering the strong electrostatic interaction between ions, we applied the Particle-Particle ParticleMesh solver with a precision value of 0.001 to calculate the longrange Coulombic forces (ESI 1.2 $\dagger$ ). The bond stretching and angle bending in molecules are described by the harmonic potential, as specified in the TIP3P water model and the OPLS-AA model for decane. The torsion interactions for decane molecules are expressed by the opls dihedral style. Sodium and chloride ions are modelled using the potential parameters reported by Lyubartsev et al. ${ }^{63}$ The force field parameters for all molecule types, including water, decane and ions, in our simulations were proven to be effective (Table S1, ESI $\dagger$ ).

\section{Simulation procedures}

The LAMMPS software ${ }^{64}$ is used to carry out the isothermalisobaric (NPT) ensemble classical MD simulations. We use the Nose-Hoover barostat and thermostat to keep the pressure and temperature balanced at 1 atm and $300 \mathrm{~K}$, respectively (ESI 1.2 $\dagger$ ).

Before running the simulations, the steepest descent algorithm style is used to minimize the system energy by adjusting the atom coordinates to avoid atom overlap. The Newtonian equations of motion are integrated by the velocity-Verlet algorithm with a time step of $1 \mathrm{fs}$. Since the transport of ions across the interface cannot occur without external forces, an electric field $E$ of $0.001,0.005$, $0.01,0.05,0.1$ and $0.5 \mathrm{~V}^{-1}$ is introduced to drive ions through the interface. In practice, these driving forces result from the attraction between ions and the surfactants in the interface or another phase. In our simulations, this electric field is only applied to ions, not to water and decane molecules. It provides a driving force for ions to transfer across the interface. By this means, the computation time and space for surfactants can be saved to improve the computing efficiency.

To ensure this condition does not affect the physical results and create biased sampling, the effects of the electric field on the simulated system are further discussed (ESI $\dagger$ Section S1.3). The total simulation time is $10 \mathrm{~ns}$. In order to reduce the storage requirements, the statistics of the simulation results are collected every 10000 timesteps for the data analysis. In addition, we do some verifications for the simulation model including the density and interfacial tension to prove our model is effective and reasonable (ESI 1.3†).

\section{Results and discussion}

\section{Ion transfer through water ridges and fingers}

We studied the effects of the electric field intensity and ionic concentrations on the transport of ions across the interface to get information on interfacial structures. Different from single ion transfer across the interface, we found different evolution processes of interfacial structures (ESI 2.1†). The results show that the transfer of ions across the interface is only controlled by the electric field applied to the ions (Fig. S3, ESI $\dagger$ ). At low electric field intensity, of $E$ below $0.1 \mathrm{~V}^{-1}$, ions are unable to transfer across the interface, which implies the existence of an interfacial barrier. Then, as the electric field intensity rises to $0.1 \mathrm{~V}^{-1}$, ions transfer across the interface and develop into a water channel in the decane phase. It is noteworthy that the water channel is not an artifact of our model and boundary conditions; a quantitative description is given in ESI 2.2. $†$ Then, as the electric field intensity rises above $0.1 \mathrm{~V}^{-1}$, the driving force generated by the electric field is large enough for each ion to easily break through the interface, inducing interfacial instability.

The results also show that, depending on the ionic concentration, two distinct modes of transfer develop. The known water finger develops at high ionic concentrations, above $0.2 \mathrm{~mol} \mathrm{~L}^{-1}$, and a new transfer mode, named a 'water ridge', develops at low ionic concentrations, of 0.1 and $0.2 \mathrm{~mol} \mathrm{~L}^{-1}$ (Fig. 2). In the water ridge, ions aggregate at the interface in an almost straight line and induce the interfacial protrusion. Then, the ion-induced interfacial protrusion gradually develops and ions cross the interface. In the water finger, a hydrated sodium ion breaks through the interface to form a chain of water molecules linked by hydrogen bonding (H-bonds). ${ }^{35,36,45,47,65}$ We obtained further insights into the transfer modes by studying the distribution of the ion density below.

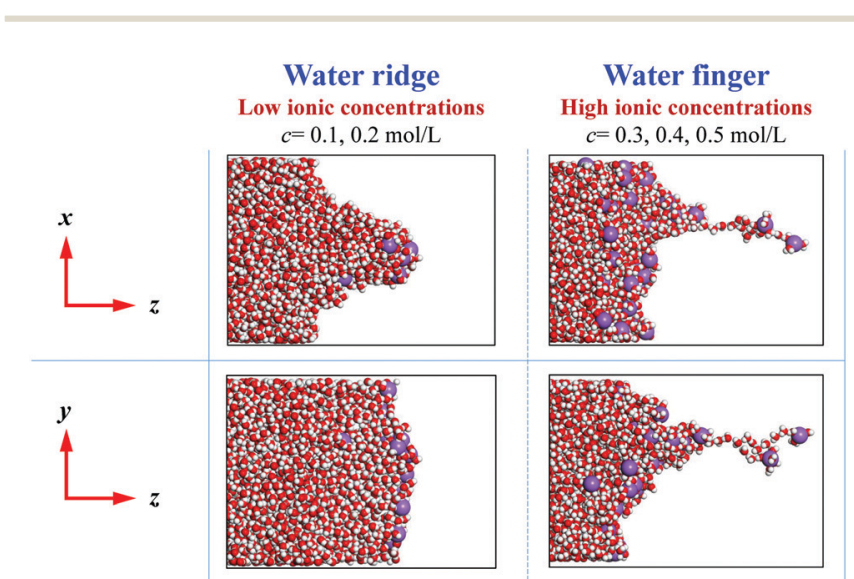

Fig. 2 Atomic view of interfacial structures during ion transfer across the interface: water ridge versus water finger. The decane molecules are set to be invisible and the sodium ions are amplified for clearer visualization. 


\section{Ion-induced interfacial protrusion}

The density distributions of molecules and the interfacial thickness under different conditions have been analyzed (ESI 2.3 and 2.4†). The density distributions of ions are presented in Fig. 3a and b. With increasing ionic concentration, more ions aggregate at the interface and the density peak rises and moves away from the decane phase. This means that the penetration depth of ions into the decane phase decreases with the ionic concentration rising. This phenomenon thus explains the changes in interfacial structures, which, in turn, induce different modes of ion transfer.

Fig. $3 c$ shows the effect of the electrical field on the $\mathrm{Na}^{+}$and $\mathrm{Cl}^{-}$densities at the interface. The results show that the peak densities increase and move toward the decane phase with the intensity of the electrical field. This finding is explained by the accumulation of sodium and chloride ions at the interface, and by deeper intrusion into the decane phase. To measure the ion intrusion depth, we use the position $\left(z_{\mathrm{o}}\right)$ where the density of decane reaches $90 \%$ of its bulk density as the reference point. The intrusion depth $\delta$ is the difference between the position where the density of ions reaches a peak $\left(z_{\mathrm{p}}\right)$ and the reference point $\left(z_{\mathrm{o}}\right)$.

Accordingly, the ion intrusion depth versus electrical field intensity and ionic concentration is displayed in Fig. 3d. The results show that the intrusion depth increases with electric field intensity. Higher variations of the intrusion depth for sodium ions are explained by the small ionic radius of $\mathrm{Na}^{+}$ versus $\mathrm{Cl}^{-}$. The results also show that the intrusion depth decreases with increasing ionic concentration. To get further information on the mechanisms, we studied hydrogen bonding and ionic interactions at the interfaces in the following section.

\section{Hydrogen bonding and ionic interactions}

We analysed $\mathrm{H}$-bonds at the interfaces using a geometric criterion, ${ }^{66}$ which is based on the length of the H-bond and
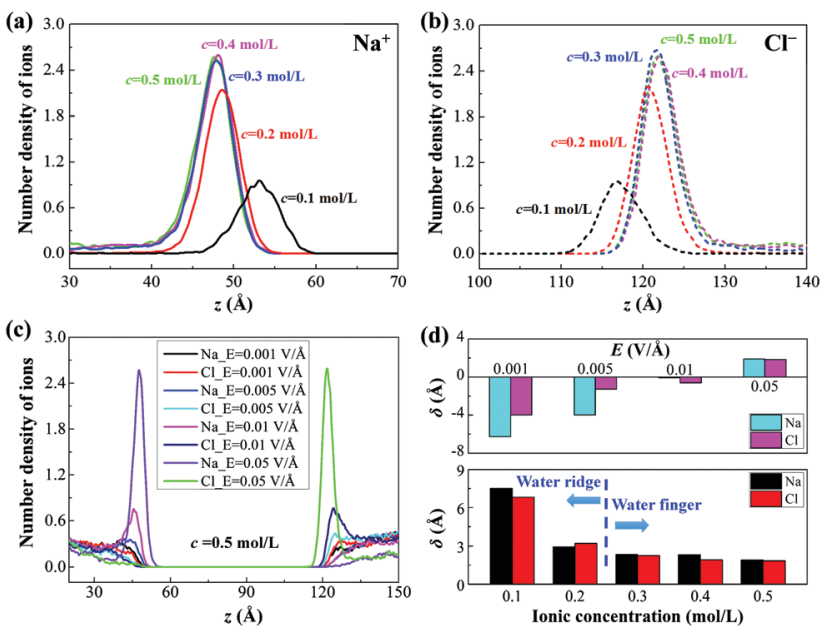

Fig. 3 Density distributions of ions along the $z$-direction under specific conditions. (a) Number density distributions of sodium ions under the $E=0.05 \vee \AA^{-1}$ condition; (b) number density distributions of chloride ions under the $E=0.05 \mathrm{~V} \AA^{-1}$ condition; (c) number density distributions of ions under the $c=0.5 \mathrm{~mol} \mathrm{~L} \mathrm{~L}^{-1}$ condition; (d) the intrusion depth under $c=$ $0.5 \mathrm{~mol} \mathrm{~L}^{-1}$ or $E=0.05 \vee \AA^{-1}$ conditions. the angle $\mathrm{H}-\mathrm{O} \cdots \mathrm{H}$. The length of the $\mathrm{H}$-bond $R_{\mathrm{OH}}$ is the length in neighbouring water molecules. We assume that $R_{\mathrm{OH}}$ is less than $3.5 \AA$ and the angle $\mathrm{H}-\mathrm{O} \cdots \mathrm{H}$ is greater than $130^{\circ}$.

The results show the number and average length of $\mathrm{H}$-bonds at the two interfaces under the same intensity of the electric field with the ion concentration rising (Fig. 4). Due to the applied electric field, sodium ions aggregate at the left interface while chloride ions accumulate at the right interface. At low ionic concentrations of 0.1 and $0.2 \mathrm{~mol} \mathrm{~L}^{-1}$, we observe a slight increase of the number of $\mathrm{H}$-bonds without a change of bond length. This finding means that the hydrogen bonding network at the interface is not broken by ion aggregation. As a consequence, the protrusion develops into a ridge resulting from a higher area and number of $\mathrm{H}$-bonds at the interface.

At higher ionic concentration $c$ above $0.2 \mathrm{~mol} \mathrm{~L}^{-1}$, the number of H-bonds decreases with ionic concentration (Fig. 4a). This phenomenon is explained by the adsorption of ions at the interface, which disturbs the directional arrangement of water molecules, and thus breaks the hydrogen bonding network. We therefore explain the two different modes of ion transfer, by a water ridge or a water finger, by whether the hydrogen bonding network at the interface is broken or not.

Fig. 4c further shows the effect of the electric field intensity on the number of $\mathrm{H}$-bonds. The results show a decrease of the $\mathrm{H}$-bond number with electric intensity, this decrease being faster for $\mathrm{Cl}^{-}$owing to its larger ionic radius. Since the hydrogen bonding network at the interfaces is significantly affected by adsorbed ions, we further analysed ion-ion interactions using the radial distribution function $(\mathrm{RDF}) g(r)$ :

$$
\begin{gathered}
g_{i j, n}(r)=\frac{\left\langle\Delta N_{i j, n}\right\rangle}{\rho_{j} V_{i j, n}} \\
V_{i j, n}=\frac{4 \pi}{3}\left(r_{n}^{3}-r_{n-1}{ }^{3}\right)
\end{gathered}
$$

where $i$ and $j$ are the central atoms and other atoms around the central atoms, $n$ represents the thin layer whose position is far away from central atom with a distance of $r$, the thickness of this layer is equal to $\left(r_{n}-r_{n-1}\right), \Delta N_{i j, n}$ is the atom number in the $n$ layer, and $\rho_{j}$ is the average number density of the $j$ atom.
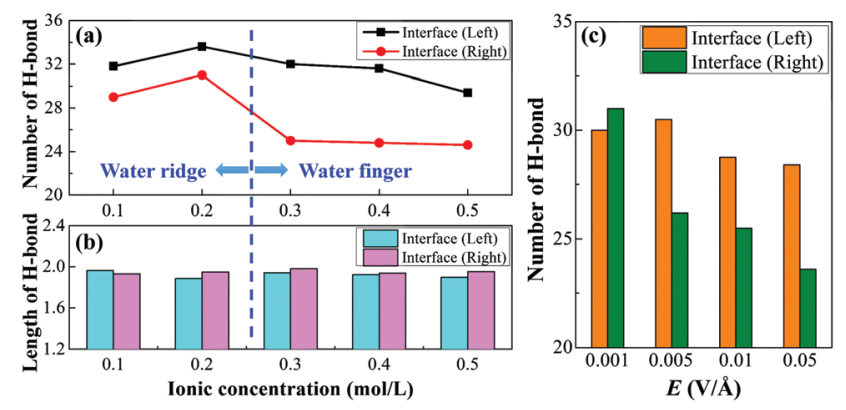

Fig. 4 Number and length of hydrogen bonds at the interface. (a) Number of hydrogen bonds at two interfaces under $E=0.05 \mathrm{~V} \AA^{-1}$ versus ionic concentration; (b) average length of hydrogen bonds at two interfaces under $E=0.05 \mathrm{~V} \AA^{-1}$ versus ionic concentration; (c) number of hydrogen bonds at two interfaces under $c=0.5 \mathrm{~mol} \mathrm{~L}^{-1}$ versus electric field intensity. 


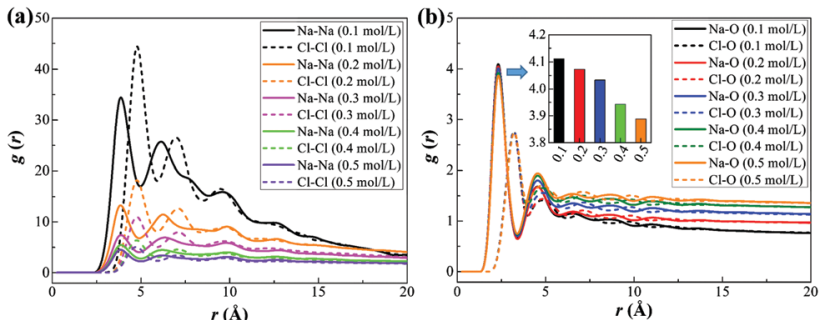

Fig. 5 Radial distribution function of ion-ion (a) and ion-water (b) versus ion concentration at the interface.

To get information on ionic interactions, we calculated $g(r)$ of $\mathrm{Na}-\mathrm{Na}, \mathrm{Cl}-\mathrm{Cl}, \mathrm{Na}-\mathrm{O}$ and $\mathrm{Cl}-\mathrm{O}$. Fig. 5 shows the densities of ions and water molecules as a function of distance from $\mathrm{Na}^{+}$and $\mathrm{Cl}^{-}$ions at the interface. The results show that the peaks decrease with the ionic concentration increasing. This trend is consistent with the change of ion transfer modes with ionic concentration. Indeed, at low concentrations, ions are regularly distributed at the interface, thus inducing a protrusion which develops into a water ridge. Whereas at higher concentrations repulsive forces induce a higher ion dispersion, which explains peak decrease and widening.

We also studied the hydration of ions at the interfaces (Fig. $5 \mathrm{~b}$ ). The larger peaks of $g(r)$ for $\mathrm{Na}-\mathrm{O}$ are explained by the stronger hydration ability of sodium ions. Moreover, the $g(r)$ peaks for $\mathrm{Na}^{+}$decrease faster than those for $\mathrm{Cl}^{-}$with increasing concentration. This finding is explained by the weaker hydration ability at high concentrations and smaller radius of sodium ions.

\section{Gibbs free energy}

The establishment of a theory for ion transport across the liquid-liquid interface is still a challenge due to the difficulty of full experimental and simulated understanding of this process. The Gibbs free energy, which can be compared with experimental data, is widely used to describe the energetic features of ion transport across the oil-water interface. Therefore, we studied the variation of the Gibbs free energy during ion transport across the interface by integrating over the $z$-directional component of the forces acting on the ions, as follows:

$$
\Delta G\left(z_{i}\right)=G\left(z_{i}\right)-G\left(z_{\text {ref }}\right)=-\int_{z_{\text {ref }}}^{z_{i}}\langle\|\vec{F}\|\rangle_{z} \mathrm{~d} z
$$

where $\langle\|\vec{F}\|\rangle_{z}$ means the averages of the $z$-directional component of the forces acting on the ions. It is noteworthy that these forces do not include the forces applied by the electric field. The $z$-direction is perpendicular to the interface. $z_{\text {ref }}$ is the reference position which corresponds to the ions in the water bulk phase. In this work, $z_{\text {ref }}$ equals $50 \AA$ and $120 \AA$ respectively for sodium and chloride ions. Although this method is not as accurate as the two coordinate method in describing the free energy due to the lack of consideration of the instantaneous interfacial structures, it is simple and can provide preliminary information of the free energy variation during ion transfer. Besides, complex interfacial structures like water ridges are also difficult to describe in the two coordinate methods.

Due to the applied electric field, sodium ions move in the direction of the electric field, while chloride ions move in the
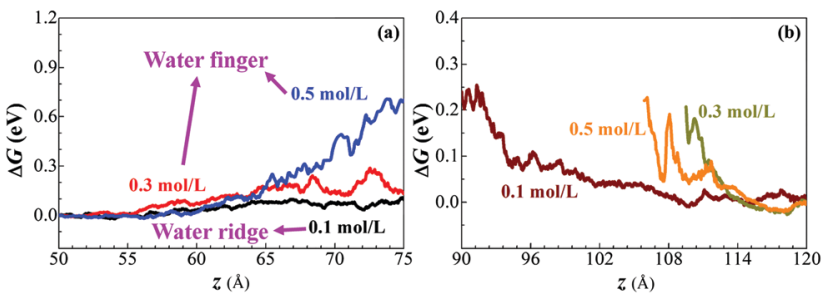

Fig. 6 Free energy of sodium ions (a) and chloride ions (b) across the decane-water interface.

opposite direction. The results show that the free energy for ion transport across the interface is rising (Fig. 6). Specifically, the variation of the free energy depends on the ionic concentrations and is directly related to the ion transfer modes.

For sodium ions, the variation ranges of the free energy increase with the ionic concentration rising. At a high ionic concentration $c$ of $0.5 \mathrm{~mol} \mathrm{~L}^{-1}$, there is a clear growth in the free energy for sodium ions. The net Gibbs free energy for the ion transfer processes is about the same order of magnitude as the results in the literature. For example, the increment of free energy for sodium ion transport from $z=50 \AA$ to $z=70 \AA$ is about $0.3 \mathrm{eV}\left(28.95 \mathrm{~kJ} \mathrm{~mol}^{-1}\right)$, which is similar to that reported for single sodium ion transport across the interface, of $29.1 \pm$ $3.3 \mathrm{~kJ} \mathrm{~mol}^{-1}{ }^{37}$ These results are thus consistent with transfer involving a water finger at high concentrations, during which the number of water molecules in the ionic hydration shells decreases significantly. ${ }^{37,38}$ Due to the difference of simulation systems, there are other factors contributing to the difference, such as the polarity of the oil phase, potential models for molecules, ionic concentration, and so on.

However, at a low ionic concentration $c$ of $0.1 \mathrm{~mol} \mathrm{~L}^{-1}$, the free energy shows very little variation (Fig. 6). We explain this phenomenon by the fact that the water ridge drags many water molecules around sodium ions into the decane phase. Therefore, the number of water molecules in the hydration shell shows few changes and thus the free energy is almost unchanged. In addition, in our simulations, the net Gibbs free energy of chloride ions (about $\left.28.95 \mathrm{~kJ} \mathrm{~mol}^{-1}\right)$ is smaller than that $\left(58.57 \pm 8.37 \mathrm{~kJ} \mathrm{~mol}^{-1}\right)$ mentioned by other literature..$^{40,43,67,68}$ We attribute this to the strong electrostatic interaction between sodium and chloride ions facilitating the transport of chloride ions across the interface.

\section{Ion transport}

The transport rate of ions has a major influence on the interfacial microstructures, thereby affecting the interfacial hydrodynamics, mass transfer and stability. Here, we studied the transport rates of ions across the interface (Fig. 7). We firstly determined the number density of ions versus ion velocity along the $z$-direction. The results show that the curves display normal distributions. Then we acquired the time-averaged transport velocities of ions across the interface after fitting these curves based on the normal distribution equation. Due to the applied electric field, sodium and chloride ions migrate in opposite directions. The stronger hydration of sodium ions means that more water molecules are tightly carried by sodium ions, inducing 

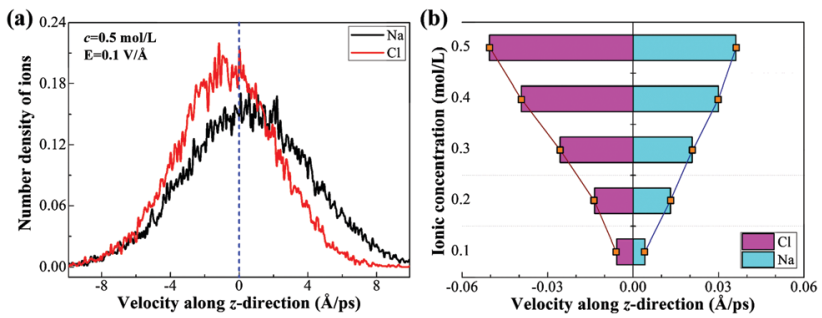

Fig. 7 Transport rate of ions in the water channel. (a) Number density distribution of ions versus velocity along the $z$-direction; (b) transport velocity along the $z$-direction of ions versus ionic concentration.

more resistance for ions across the interface. Hence, the transport velocities of $\mathrm{Na}^{+}$are lower than those for $\mathrm{Cl}^{-}$. Particularly, the velocities of ion transport across the interface increase with ionic concentration. This finding is explained by the weaker hydration ability of ions with increasing ionic concentration.

\section{Conclusion}

We studied the transport of ions across the decane-water interface using classical MD simulations. The results reveal a new mode of ion transfer involving a water ridge at low ionic concentrations, besides the known water finger mode at high ionic concentrations. These two modes operate by different mechanisms at the molecular level. In the water ridge, the sparse distribution of ions at the interface does not break the hydrogen bonding network and thus induces a regular, large protrusion named a water ridge. Whereas at high concentrations, both higher ion aggregation and repulsive interactions break the hydrogen bonding network, thus inducing the transfer of single, hydrated ions through a water finger. Meanwhile, the Gibbs free energy change of ion transport across the interface is dependent on the ionic concentration. The water ridge at low concentrations, which drags more water molecules around sodium ions into the decane phase, experiences a smaller free energy change than the water finger at high concentrations. In addition, the velocity of ion transport is positively related with the ionic concentration.

Our discovery of the water ridge has implications in all biological, chemical and physical processes involving ion transport across the interface at low ionic concentrations. Different from the water finger, the water ridge can induce more distinct interfacial deformation and water dragging effects of ions, therefore reducing the free energy variation and facilitating interfacial mass transfer. In order to deeply reveal the hidden mechanism of water ridges, more comprehensive investigations of the 2D free energy surface based on the two coordinate method considering instantaneous interfacial structures and ionic hydration are necessary. We are now further investigating the transfer mechanism of multiple ions in detail, involving the role of interfacial deformation and ionic hydration using the umbrella sampling method.

\section{Conflicts of interest}

There are no conflicts to declare.

\section{Acknowledgements}

This work was supported by the China National Funds for Distinguished Young Scientists (Grant No. 51425603).

\section{References}

1 H. Deng, J. E. Dick, S. Kummer, U. Kragl, S. H. Strauss and A. J. Bard, Anal. Chem., 2016, 88, 7754-7761.

2 L. J. Sanchez Vallejo, J. M. Ovejero, R. A. Fernández and S. A. Dassie, Int. J. Electrochem., 2012, 1-34.

3 B. Wei, R. Wu, L. Lu, X. Ning, X. Xu, C. Wood and Y. Yang, Energy Fuels, 2017, 31, 12035-12045.

4 N. Schelero and R. von Klitzing, Soft Matter, 2011, 7, 2936-2942.

5 G. Chevrot, R. Schurhammer and G. Wipff, Phys. Chem. Chem. Phys., 2007, 9, 1991-2003.

6 M. M. Nouamane Laanait, B. Hou, H. Yu, P. Vanýsek, M. Meron, B. Lin, I. Benjamin and M. L. Schlossman, Proc. Natl. Acad. Sci. U. S. A., 2012, 109, 20326-20331.

7 Y. Li, M. Girard, M. Shen, J. A. Millan and M. Olvera de la Cruz, Proc. Natl. Acad. Sci. U. S. A., 2017, 114, 11838-11843.

8 K. E. Wardle, D. J. Henderson and R. L. Rowley, Fluid Phase Equilib., 2005, 233, 96-102.

9 E. J. Robertson, A. P. Carpenter, C. M. Olson, R. K. Ciszewski and G. L. Richmond, J. Phys. Chem. C, 2014, 118, 15260-15273.

10 X. Hua, J. Frechette and M. A. Bevan, Soft Matter, 2018, 14, 3818-3828.

11 W. Gan, W. Wu, F. Yang, D. Hu, H. Fang, Z. Lan and Q. Yuan, Soft Matter, 2017, 13, 7962-7968.

12 H. Fang, W. Wu, Y. Sang, S. Chen, X. Zhu, L. Zhang, Y. Niu and W. Gan, RSC Adv., 2015, 5, 23578-23585.

13 J. C. Newland, P. R. Unwin and J. V. Macpherson, Phys. Chem. Chem. Phys., 2014, 16, 10456-10463.

14 Z. Liang, W. Bu, K. J. Schweighofer, D. J. Walwark, J. S. Harvey, G. R. Hanlon, D. Amoanu, C. Erol, I. Benjamin and M. L. Schlossman, Proc. Natl. Acad. Sci. U. S. A., 2018, 201701389.

15 E. W. E. Scoppola, G. Li Destri, L. Porcar, R. A. Campbell, O. Konovalov, G. Fragneto and O. Diat, Phys. Chem. Chem. Phys., 2015, 17, 15093-15097.

16 T. Wandlowski, V. Mareček, Z. Samec and R. Fuoco, J. Electroanal. Chem., 1992, 331, 765-782.

17 Z. Samec, Electrochim. Acta, 1998, 44, 85-90.

18 T. K. And and Y. Takasu, J. Phys. Chem. B, 1997, 101, 5963-5968.

19 T. R. Underwood and H. C. Greenwell, Sci. Rep., 2018, 88, 352 .

20 C. L. McFearin and G. L. Richmond, J. Phys. Chem. C, 2009, 113, 21162-21168.

21 C. D. Wick, T.-M. Chang, J. A. Slocum and O. T. Cummings, J. Phys. Chem. C, 2012, 116, 783-790.

22 C. Sun, M. Liu and B. Bai, Carbon, 2019, 153, 481-494.

23 C. Sun, S. Zhu, M. Liu, S. Shen and B. Bai, J. Phys. Chem. Lett., 2019, 10, 7188-7194.

24 C. D. Wick and S. S. Xantheas, J. Phys. Chem. B, 2009, 113, 4141-4146.

25 C. D. Wick and L. X. Dang, Chem. Phys. Lett., 2008, 458, 1-5. 
26 C. D. Wick and O. T. Cummings, Chem. Phys. Lett., 2011, 513, 161-166.

27 M. X. Luo, G. K. Olivier and J. Frechette, Soft Matter, 2012, 8, 11923-11932.

28 G. I. Guerrero-Garcia, Y. F. Jing and M. O. de la Cruz, Soft Matter, 2013, 9, 6046-6052.

29 P. Sun, K. Huang and H. Liu, Soft Matter, 2019, 15, 4346-4350.

30 D. Bastos-González, L. Pérez-Fuentes, C. Drummond and J. Faraudo, Curr. Opin. Colloid Interface Sci., 2016, 23, 19-28.

31 J. Zwanikken and R. van Roij, Phys. Rev. Lett., 2007, 99, 178301.

32 B. Wen, C. Sun, B. Bai, E. Y. Gatapova and O. A. Kabov, Phys. Chem. Chem. Phys., 2017, 19, 14606-14614.

33 B. Wen, C. Sun and B. Bai, Phys. Chem. Chem. Phys., 2018, 20, 22796-22804.

34 G. Luo, S. Malkova, J. Yoon, D. G. Schultz, B. Lin, M. Meron, I. Benjamin, P. Vanýsek and M. L. Schlossman, Science, 2006, 311, 216-218.

35 I. Benjamin, Science, 1993, 261, 1558-1560.

36 L. X. Dang, J. Phys. Chem. B, 1999, 103, 8195-8200.

37 P. A. Fernandes, M. N. D. S. Cordeiro and J. A. N. F. Gomes, J. Phys. Chem. B, 2000, 104, 2278-2286.

38 D. J. V. A. dos Santos and J. A. N. F. Gomes, ChemPhysChem, 2002, 3, 946-951.

39 I. Chorny and I. Benjamin, J. Phys. Chem. B, 2005, 109, 16455-16462.

40 I. Benjamin, J. Phys. Chem. B, 2013, 117, 4325-4331.

41 J. J. Karnes and I. Benjamin, J. Chem. Phys., 2016, 145, 014701.

42 P. A. Fernandes, M. N. D. S. Cordeiro and J. A. N. F. Gomes, J. Phys. Chem. B, 2000, 104, 2278-2286.

43 K. J. Schweighofer and I. Benjamin, J. Phys. Chem., 1995, 99, 9974-9985.

44 A. A. Kornyshev, A. M. Kuznetsov and M. Urbakh, J. Chem. Phys., 2002, 117, 6766-6779.

45 N. Kikkawa, L. Wang and A. Morita, J. Am. Chem. Soc., 2015, 137, 8022-8025.

46 Y. Tabe, N. Kikkawa, H. Takahashi and A. Morita, J. Phys. Chem. C, 2014, 118, 977-988.

47 N. Kikkawa, L. Wang and A. Morita, J. Chem. Phys., 2016, 145, 014702.
48 L. Wang, N. Kikkawa and A. Morita, J. Phys. Chem. B, 2018, 122, 3562-3571.

49 N. Kikkawa, T. Ishiyama and A. Morita, Chem. Phys. Lett., 2012, 534, 19-22.

50 N. J. Hadia, T. Hansen, M. T. Tweheyo and O. Torsæter, Energy Fuels, 2012, 26, 4328-4335.

51 B. Qiao, J. V. Muntean, M. Olvera de la Cruz and R. J. Ellis, Langmuir, 2017, 33, 6135-6142.

52 S. C. Ou, D. Cui and S. Patel, Phys. Chem. Chem. Phys., 2016, 18, 30357-30365.

53 J. Tang, Z. Qu, J. Luo, L. He, P. Wang, P. Zhang, X. Tang, Y. Pei, B. Ding, B. Peng and Y. Huang, J. Phys. Chem. B, 2018, 122, 1905-1918.

54 H. Monteillet, F. Hagemans and J. Sprakel, Soft Matter, 2013, 9, 11270-11275.

55 R. H. Swendsen and J. S. Wang, Phys. Rev. Lett., 1986, 57, 2607-2609.

56 D. Hamelberg, J. Mongan and J. A. McCammon, J. Chem. Phys., 2004, 120, 11919-11929.

57 G. M. Torrie and J. P. Valleau, J. Comput. Phys., 1977, 23, 187-199.

58 A. Barducci, G. Bussi and M. Parrinello, Phys. Rev. Lett., 2008, 100, 020603.

59 W. Wan, J. Zhao, J. H. Harwell and B.-J. Shiau, J. Dispersion Sci. Technol., 2016, 37, 280-287.

60 S. L. Price, A. J. Stone and M. Alderton, Mol. Phys., 1984, 52, 987-1001.

61 W. L. Jorgensen, J. Chandrasekhar, J. D. Madura, R. W. Impey and M. L. Klein, J. Chem. Phys., 1983, 79, 926-935.

62 S. W. I. Siu, K. Pluhackova and R. A. Böckmann, J. Chem. Theory Comput., 2012, 8, 1459-1470.

63 A. P. Lyubartsev and A. Laaksonen, J. Phys. Chem., 1996, 100, 16410-16418.

64 S. Plimpton, P. Crozier and A. Thompson, J. Comput. Phys., 1995, 117, 1-19.

65 I. Benjamin, J. Chem. Phys., 1992, 97, 1432-1445.

66 R. Kumar, J. R. Schmidt and J. L. Skinner, J. Chem. Phys., 2007, 126, 05B611.

67 L. X. Dang, J. Phys. Chem. B, 2001, 105, 804-809.

68 Y. Marcus, Pure Appl. Chem., 1990, 62, 2069-2076. 


\section{Supplementary Information}

\section{Evidence for water ridges at oil-water interfaces: implications for ion transport}

Boyao Wen, Chengzhen Sun, Wenxiu Zheng, Bofeng Bai * and Eric Lichtfouse

State Key Laboratory of Multiphase Flow in Power Engineering, Xi'an Jiaotong University, Xi'an, Shaanxi 710049, China

* Author to whom correspondence should be addressed. Electronic mail: bfbai@mail.xjtu.edu.cn 


\section{Contents}

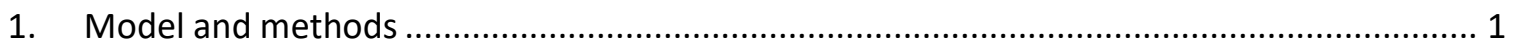

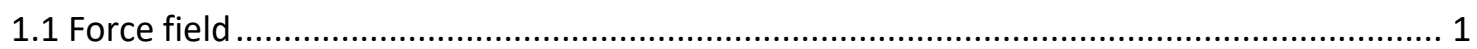

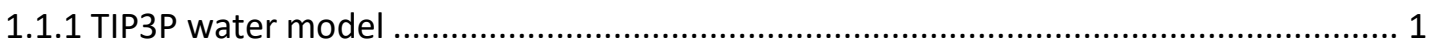

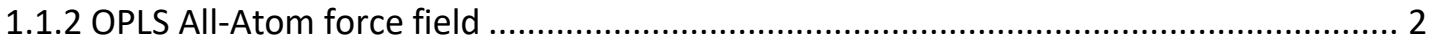

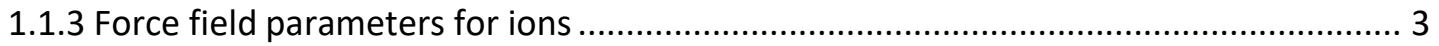

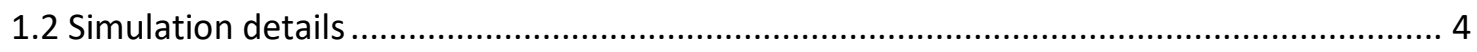

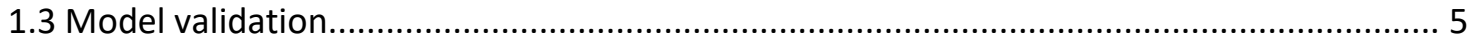

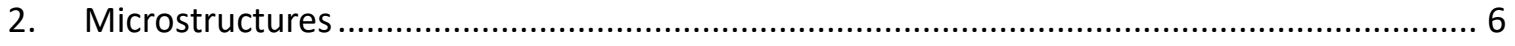

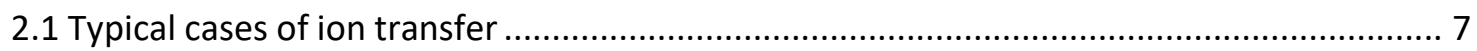

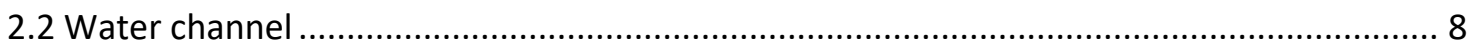

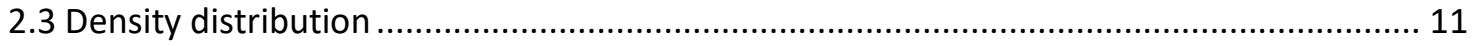

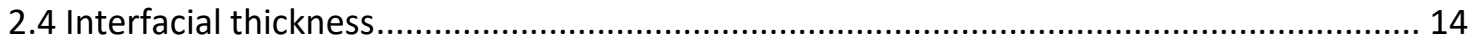

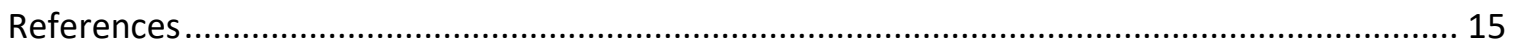




\section{Model and methods}

\subsection{Force field}

\subsubsection{TIP3P water model}

As the most important substance on Earth, water plays a key role in many biological, chemical, physical and industrial processes. With the development of computer simulation techniques, developing an accurate water model to predict the properties of water is an important subject of research. The complexity of the water properties combined with multiple possible approximation (e.g., quantum vs. classical, flexible vs. rigid) has led to the proposal of many computational models for water. ${ }^{1-8}$ However, it is very difficult to choose the best potential model of water because every water potential model is often developed and applied for describing a specific property. Because of our regular simulation conditions, we choose TIP3P water model with a long-range Coulombic solver considering the effect of ions to simulate water molecules. In our previous work, ${ }^{9,10}$ we showed that this water model can accurately simulate the density and structures of water molecules at the interfaces. Meanwhile, the interfacial tension between decane and water phase is consistent with experimental values, reflecting the nice combination of decane potential and water model. In this study, we employ the TIP3P model, ${ }^{11}$ which is proven to be efficient and can precisely predict the structure and dynamics of the water phase, to simulate the interactions between water molecules. The TIP3P model assigns the charge and LJ parameter for each atom of the water molecule. Additionally, the harmonic bond and angle style are utilized to describe the O$\mathrm{H}$ bond and the H-O-H angle. In 1984, Price et al. ${ }^{12}$ modified the parameters and developed the TIP3P model with a long-range Coulomnic solver, which can effectively 
describe electrostatic interaction. The simulation parameters in our work are identical with those parameters modified by Price et al. ${ }^{12}$ More detailed parameters can be found in Table S1.

\subsubsection{OPLS All-Atom force field}

In this paper, the OPLS All-Atom (OPLS-AA) force field ${ }^{13}$ is utilized to mimic the interactions between decane molecules. Our previous simulation results prove its ability to accurately reproduce the thermodynamic and interfacial properties of decane, such as density, diffusion coefficients and interfacial tension. ${ }^{9,10}$ The non-bonded interactions, bond stretching, angle bending and torsion interactions are considered in this force field:

$$
E=E_{a b}+E_{\text {bond }}+E_{\text {angle }}+E(\phi)
$$

The non-bonded interaction includes the standard 12/6 Lennard-Jones and Coulombic pairwise interactions, given by

$$
\left.E_{a b}=4 \varepsilon\left(\frac{\sigma}{r_{a b}}\right)^{12}-\left(\frac{\sigma}{r_{a b}}\right)^{6}\right]+\frac{C q_{a} q_{b}}{\chi r_{a b}} \quad\left(r_{a b}<r_{c u t}\right)
$$

where $\varepsilon$ is the depth of the potential well, $\sigma$ is the distance between atoms when the potential is zero, $r_{\text {cut }}$ is the cutoff radius, $C$ is an energy-conversion constant, $q_{a}$ and $q_{b}$ are the charges of atom $a$ and $b, \chi$ is the dielectric constant. While $r_{a b}$ is greater than $r_{c u t}$, we only calculate the long-range electrostatic interactions by using the Particle-Particle Particle-Mesh (PPPM) method. Potential parameters between unlike atoms are obtained based on the Lorentz-Berthelot combining rule:

$$
\sigma_{i j}=\frac{\sigma_{i i}+\sigma_{j j}}{2} \quad \varepsilon_{i j}=\sqrt{\varepsilon_{i i} \varepsilon_{j j}}
$$

The bond stretching and angle bending in the molecule are described by the harmonic bond style, as follows: 


$$
\begin{aligned}
& E_{\text {bond }}=K_{\text {bond }}\left(r_{a b}-r_{0}\right)^{2} \\
& E_{\text {angle }}=K_{\text {angle }}\left(\theta-\theta_{0}\right)^{2}
\end{aligned}
$$

where $K_{\text {bond }}$ and $K_{\text {angle }}$ are the energy-related bond and angle coefficients, $r_{0}$ is the equilibrium bond distance, $\theta_{0}$ is the equilibrium angle value.

The last term of the OPLS-AA force field is the torsion interaction, which is described by the opls dihedral style, as follows:

$$
E(\phi)=\frac{V_{1}}{2}[1+\cos (\phi)]+\frac{V_{2}}{2}[1-\cos (2 \phi)]+\frac{V_{3}}{2}[1+\cos (3 \phi)]
$$

where $V_{1}, V_{2}$ and $V_{3}$ are the dihedral coefficients. The detailed parameters of OPLS-AA potential for decane molecules can be found in Table S1.

\subsubsection{Force field parameters for ions}

The potential parameters for sodium/chloride ions we used are the parameters reported by Lyubartsev et al., ${ }^{14}$ which are proved to be accurate and effective for simulating the

\begin{tabular}{|c|c|c|c|}
\hline \multicolumn{4}{|c|}{ Water (TIP3P model) ${ }^{11,12}$} \\
\hline L-J potential parameters & $\sigma(\AA)$ & $\varepsilon\left(10^{-3} \mathrm{eV}\right)$ & charge $(\mathrm{e})$ \\
\hline $\mathrm{O}$ & 3.188 & 4.423 & -0.830 \\
\hline $\mathrm{H}$ & 0 & 0 & 0.415 \\
\hline Bond potential parameters & \multicolumn{2}{|c|}{$K_{\text {bond }}\left(10^{2} \mathrm{eV} \cdot \AA^{-2}\right)$} & $r_{0}(\AA)$ \\
\hline $\mathrm{O}-\mathrm{H}$ & \multicolumn{2}{|c|}{19.514} & 0.9572 \\
\hline Angle potential parameters & \multicolumn{2}{|c|}{$K_{\text {angle }}\left(\mathrm{eV} \cdot \mathrm{rad}^{-2}\right)$} & $\theta_{0}\left({ }^{\circ}\right)$ \\
\hline $\mathrm{H}-\mathrm{O}-\mathrm{H}$ & \multicolumn{2}{|c|}{2.385} & 104.52 \\
\hline \multicolumn{4}{|c|}{ Decane (OPLS-AA model) $^{13}$} \\
\hline L-J potential parameters & $\sigma(\AA)$ & $\varepsilon\left(10^{-3} \mathrm{eV}\right)$ & charge $(\mathrm{e})$ \\
\hline $\mathrm{C}, \mathrm{RCH}_{3}$ & 3.500 & 2.862 & -0.180 \\
\hline $\mathrm{C}, \mathrm{R}_{2} \mathrm{CH}_{2}$ & 3.500 & 2.862 & -0.120 \\
\hline $\mathrm{H}$ & 2.500 & 1.301 & 0.060 \\
\hline
\end{tabular}
structure and the dynamical properties of ions in the water phase.

Table S1. Force field parameters for water, decane and ions. 


\begin{tabular}{|c|c|c|c|}
\hline Bond potential parameters & $K_{\text {bond }}\left(10^{2} \mathrm{eV} \cdot \AA^{-2}\right)$ & & $r_{0}(\AA)$ \\
\hline C-C & 13.454 & & 1.526 \\
\hline $\mathrm{C}-\mathrm{H}$ & 14.365 & & 1.090 \\
\hline Angle potential parameters & $K_{\text {angle }}\left(\mathrm{eV} \cdot \mathrm{rad}^{-2}\right)$ & & $\theta_{0}\left({ }^{\circ}\right)$ \\
\hline H-C-H & 1.518 & & 104.52 \\
\hline $\mathrm{H}-\mathrm{C}-\mathrm{C}$ & 1.518 & & 109.50 \\
\hline $\mathrm{C}-\mathrm{C}-\mathrm{C}$ & 1.736 & & 109.50 \\
\hline Dihedral potential parameters & $V_{1}(\mathrm{eV})$ & $V_{2}(\mathrm{eV})$ & $V_{3}(\mathrm{eV})$ \\
\hline C-C-C-C & 0.0755 & -0.0068 & 0.0121 \\
\hline H-C-C-C & 0 & 0 & 0.0159 \\
\hline $\mathrm{H}-\mathrm{C}-\mathrm{C}-\mathrm{H}$ & 0 & 0 & 0.0138 \\
\hline \multicolumn{4}{|c|}{ Ions ${ }^{14}$} \\
\hline L-J potential parameters & $\sigma(\AA)$ & $\left(10^{-3} \mathrm{eV}\right)$ & charge (e) \\
\hline $\mathrm{Na}^{+}$ & 2.586 & 3.706 & 1 \\
\hline $\mathrm{Cl}^{-}$ & 4.4015 & 1.740 & -1 \\
\hline
\end{tabular}

\subsection{Simulation details}

In this paper, the Nose-Hoover style non-Hamiltonian equations of motion ${ }^{15,16}$ are used to generate positions and velocities of atoms in the simulated system from the NPT ensembles. The equations of motion used in the simulations are those of Shinoda et al., ${ }^{17}$ which combine the hydrostatic equations of Martyna et al. ${ }^{18}$ with the strain energy proposed by Parrinello and Rahman. ${ }^{19}$ The time integration schemes closely follow the time-reversible measure-preserving Verlet and IRESPA integrators derived by Tuckerman et al. ${ }^{20}$ The $T_{\text {damp }}$ parameter which determines how quickly the temperature relaxed is a value of $0.2 \mathrm{~K}$, while the $P_{\text {damp }}$ deciding the time scale on which pressure relaxed is a value of $1 \mathrm{ps}$. It is noteworthy that the good choice of the value of $P_{\text {damp }}$ is about 1000 timesteps. If it is too small, the pressure and volume fluctuate severely; if it is too long, the equilibrium time for pressure is very long. In order to reduce the storage 
requirements for the data analysis, the coordinates of the ions and molecules are output every 10000 timesteps, i.e. 1 ps.

Because of the charge properties of ions, the long-range Coulombic interactions between ions or molecules play an important role in the simulation systems. Thus, a solver to compute the long-range Coulombic interactions needs to be defined. In our simulations, we adopt the PPPM solver to calculate the long-range Coulombic force. This method uses 3d FFTs to solve Poisson's equation on the mesh which atom charge is mapped, then interpolates electric fields on the mesh points back to the atoms. The PPPM solver is a faster method to reduce the computation time and memory storage, because it scales as $\mathrm{N} \log (\mathrm{N})$ where $\mathrm{N}$ is the total atom numbers, which is far less than the Ewald summation $\left(\mathrm{N}^{\wedge}(3 / 2)\right)$. The accuracy of PPPM method is $0.001 \mathrm{eV} / \AA$ and the grid of the mesh is $10 \times 10 \times 30$ in the simulations. In our previous work, ${ }^{10}$ we had compared a serials of number of grid mesh and verify that this grid size is rational to calculate the electrostatic interaction and improve computing efficiency.

\subsection{Model validation}

In order to illustrate the reasonableness and reliability of the simulation model and potential parameters, we had made a validation by comparing the simulated bulk densities or interfacial tension (IFT) with the actual value of them under the same conditions in our previous work. ${ }^{9,10}$ Therefore, we do not repeat it here. Different from previous models, we introduce an electric field which only applies external force on the ions to simulate the driving forces like surfactants for ions to transport across the interfaces. It is necessary to provide a discussion of the effects of electric field on the 
simulation models. Figure S1 shows the effects of electric field on the energies (kinetic energy, potential energy, E_coul and E_vdwl) of simulation system.

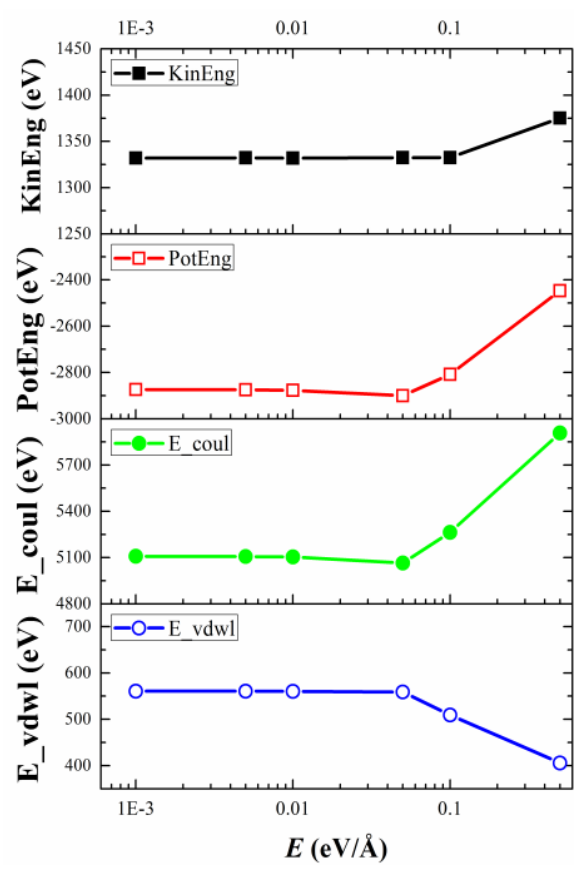

Figure S1. Variation of energies versus electric field intensity

As we can see from Figure S1, when the intensity of electric field $E$ is smaller than $0.1 \mathrm{eV} / \AA$, the variation of different energies is almost unchanged, which means that the electric field does not creates an unphysical system and the effects of electric field on the simulation results can be ignored. For specific electric field $(E=0.1 \mathrm{eV} / \AA$ ) , the formation of water channel in the decane phase may induces a little change of the energies. However, the system is stable and the effects of electric field is also small. When $E=0.5$ eV/Å, single ions can easily transfer across the decane-water interfaces and finally break the stable interface systems. Therefore, the energies of systems under this electric field intensity show significant change.

\section{Microstructures}




\subsection{Typical cases of ion transfer}

In our simulations, we studied the ions transfer processes involving multiple ions at interfaces. Different from the case of single ions transfer across the interface, we found different evolution processes of the interfacial structures. Figure $\mathbf{S 2}$ shows several typical cases during ions transfer across the interfaces under different ionic concentrations. At low ionic concentrations, a specific "water ridge" interfacial structure is found (Case 1), rather than the water finger. At high concentrations, different cases may occur. For Case 2 , individual ion with a hydration shell transfer through the interface like the water finger of single ion across the interface. For Case 3, due to the interaction between ions at the interfaces, several ions successively transfer across the interface and form a more stable water finger with strong connectivity of water molecules.

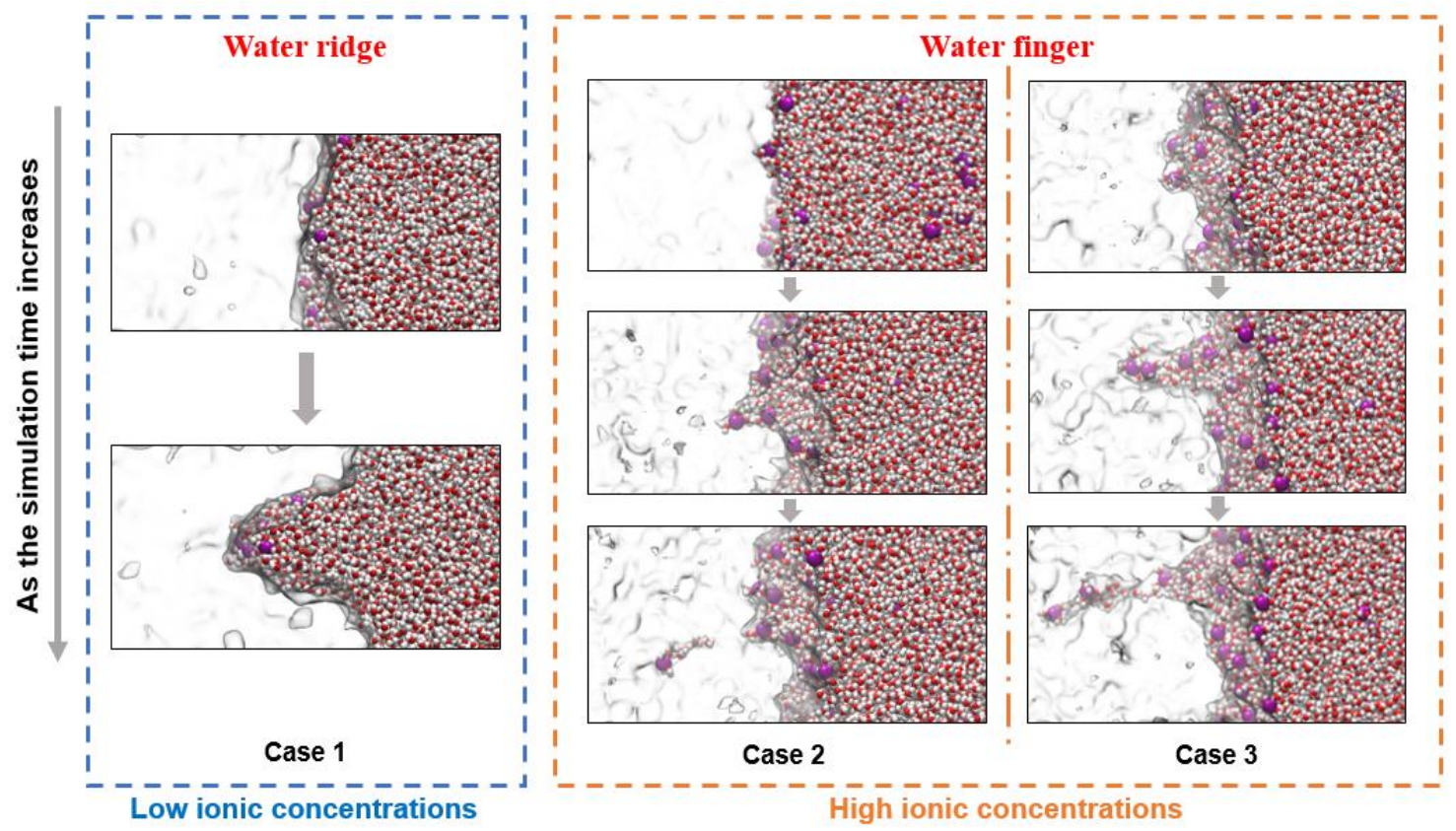

Figure S2. Different cases of ions transfer. (The glassblowing color: decane phase; purple: sodium ions; red: oxygen atoms; white: hydrogen atoms) 


\subsection{Water channel}

Figure $\mathbf{S 3}$ shows the diagram of transport behaviors of ions across the decane-water interface under the effects of ionic concentrations and external forces. The transfer behaviors of ions across the interfaces are only related to the electric field applied on the ions. With the electric field intensity increasing, the sodium and chloride ions are separated gradually in the opposite direction and deposited at two decane-water interface separately. For low electric field intensity $(E<0.1 \mathrm{~V} / \AA ̊$ ), ions are unable to transfer across the interfaces because the driving force generated by electric field cannot break through the barrier of interfaces. Particularly, the electric field $(E<0.01 \mathrm{~V} / \AA ̊$ ) force even cannot separate the ion-pair formed by sodium and chloride ions. As the electric field intensity rises $(E \geq 0.1 \mathrm{~V} / \AA ̊ \AA)$, ions transport across the decane-water interface but only develop into a water channel in the decane phase under specific electric field intensity ( $E$ < $0.1 \mathrm{~V} / \AA$ A). The transfer modes and mechanism of ions across the interfaces are detailedly analyzed in the manuscript.

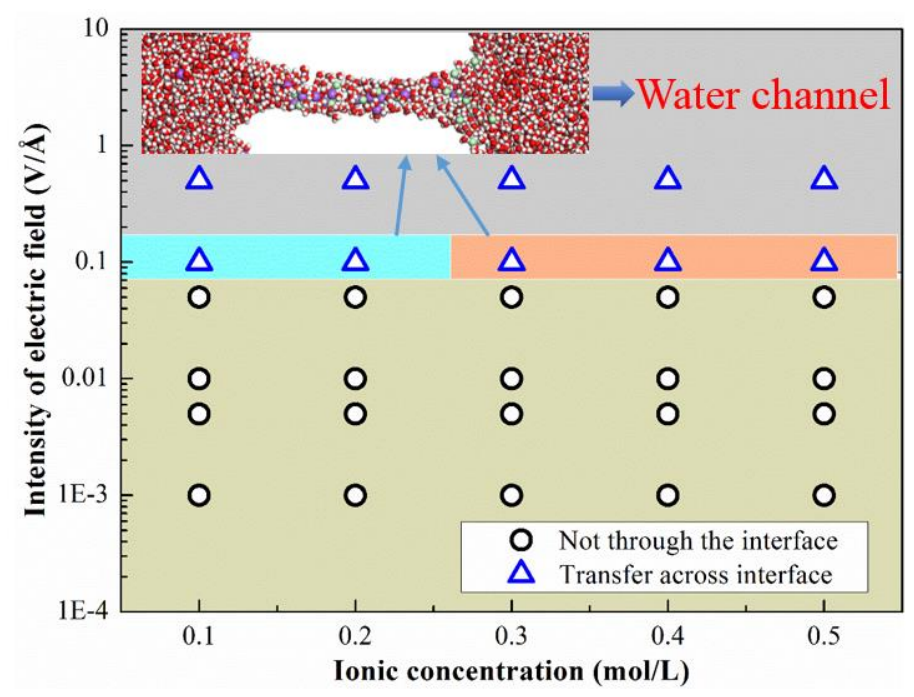

Figure S3. Phase diagram of ions transport across the interface and atomic view of water channel 
Owing to the immiscibility between ions and decane phase, ions transfer across the interface and penetrate through the decane phase with the form of water channel, which is also mentioned in other literatures. Different from our "sandwich" simulation model, Qiao et al. ${ }^{21}$ formulated a water-in-oil microemulsion model, in which a $5 \mathrm{~nm}$ diameter water is surrounded by oil molecules and ions are transported from water to the oil phase under the effect of extractants. In addition, Tang et al. ${ }^{22}$ studied the detachment of a situated oil layer from the hydroxylated silica surface and found the water channel formation when the surfactants penetrate into the oil layer under the strong polarinteraction between surfactants and silica substrates. Figure $\mathbf{S 4}$ shows the comparison of these simulation with our model. We may conclude that the formation of water channel is a specific phenomenon which has nothing to do with the simulation model and conditions, such as model size, boundary conditions, among others.

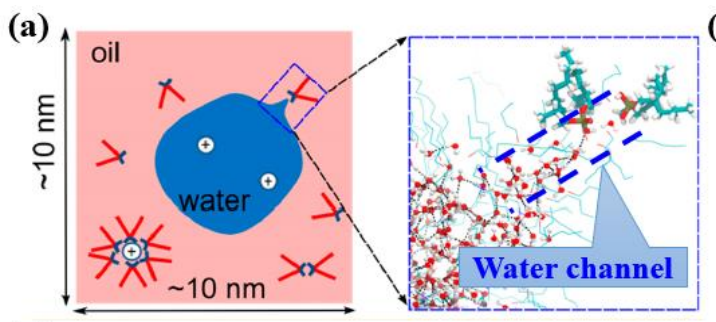

(b)

(c)
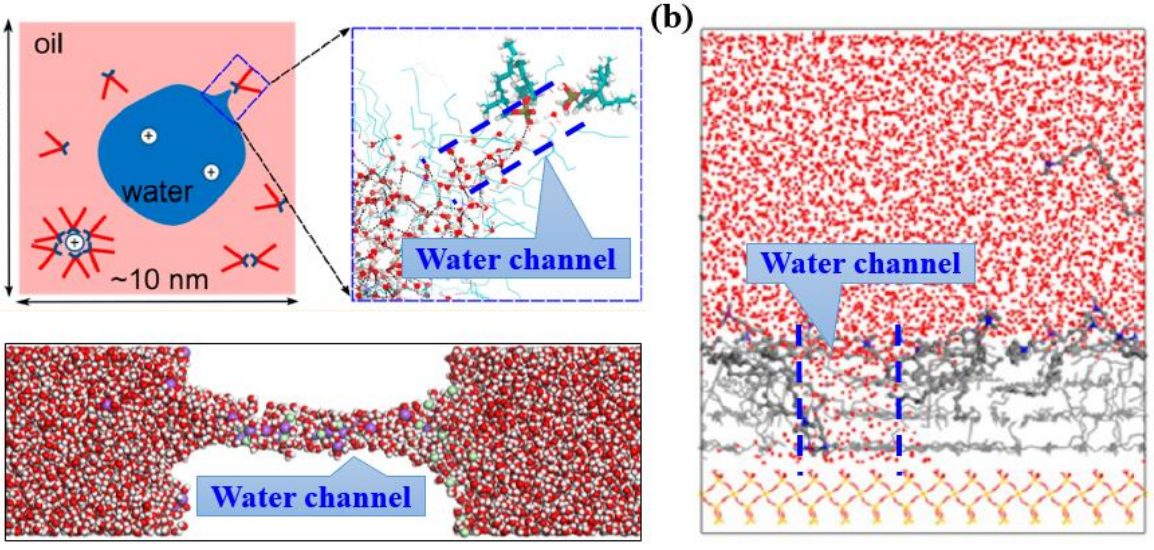

Figure S4. Comparison of simulation models. (a) Water-in-oil microemulsion model, from Ref. [13]; (b) Oil layer model at the solid surface, from Ref. [14]; (c) Our model.

Further, we give some quantitative description of the water channel, as shown in Figure S5. The diameter of water channel increases with ionic concentration rising. In order to analyze how characteristics of water channel change with ionic concentration, 
we propose two geometric parameters: $D$ and $D^{\prime}$, which stand for diameters of water channel and ion distribution area respectively (see Figure S5(b)). Since the size of water channel is not uniform across the decane phase and fluctuates as a function of time, these parameters are obtained by calculating the time-averaged diameters of water channel along $z$-direction. The maximum diameter of $\mathrm{O}$ and $\mathrm{H}$ atoms distributing at the water channel is named $D$ while the maximum diameter of ions distributing at the water channel is called $D^{\prime}$. The time-averaged percentage distributions of atoms at water channel show that the ions tend to distribute at the central region of water channel because of their hydration. Figure S5(c) displays the $D$ and $D-D^{\prime}$ versus ionic concentration. With ionic concentration increasing, the diameter of water channel $D$ increases sharply and then tends to a certain value while the value of $D-D^{\prime}$ shows upward trend with a characteristic stage. At low ionic concentration $(c=0.1$ and $0.2 \mathrm{~mol} / \mathrm{L})$, the values of $D-D^{\prime}$ are roughly equal to $5 \sim 6 \AA$, which are about twice the diameter of water molecule $(\approx 3 \AA)$. It means that there is at least one layer of water molecules between the decane phase and ions at the water channel. At high ionic concentrations $(c>0.2 \mathrm{~mol} / \mathrm{L})$, the values of $D-D^{\prime}$ are approximately $8 \sim 9 \AA$, which are about three times the diameters of water molecule due to more water molecules carried by ions. We attribute the variation of $D-D^{\prime}$ to the change of water molecule conformation at water channel near the decane phase. Figure S5(d) compares the water conformation at $c=0.1 \mathrm{~mol} / \mathrm{L}$ and $c=0.5 \mathrm{~mol} / \mathrm{L}$. The parameter $\theta$, which is the angle between interface and direction of the water dipole moment, is adopted to describe the water conformation. At low ionic concentrations, more water molecules are perpendicular to the interface. With ionic concentration 
increasing, a weakening trend of water molecules perpendicular to the interface occurs. The distribution of angle $\theta$ is more dispersed, inducing an increase of the thickness $D-D^{\prime}$.

(a)

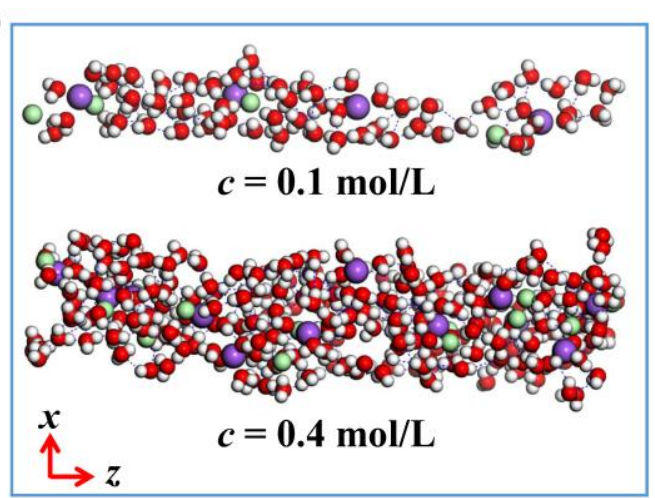

(c)

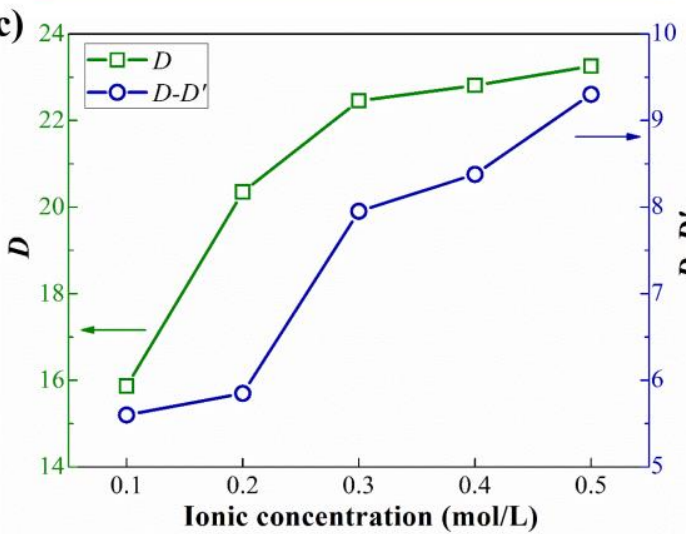

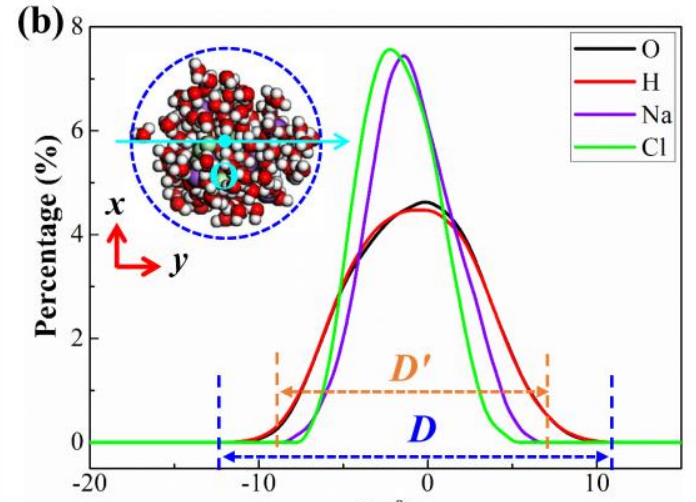

(d)

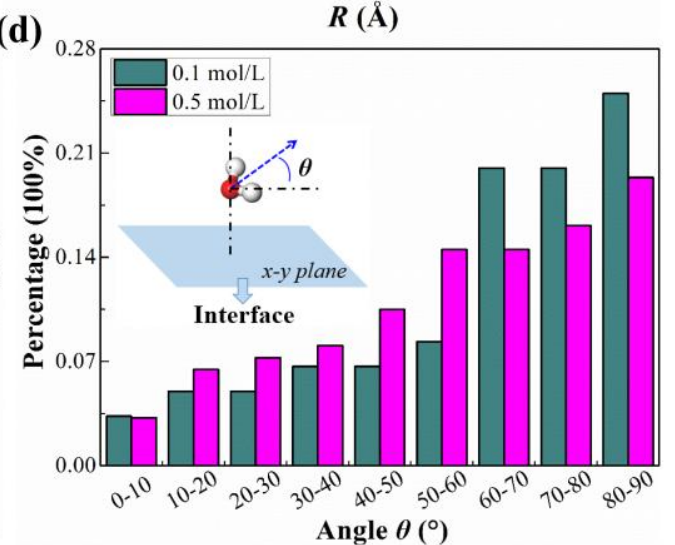

Figure S5. Characteristics of water channel. (a) Atomic views of water channel ( $c=0.1$ and $0.4 \mathrm{~mol} / \mathrm{L}$ ); (b) Percentage distributions of atoms along the radial direction at water channel ( $c=0.5 \mathrm{~mol} / \mathrm{L}$ ); (c) $D$ and $D^{-} D^{\prime}$ versus ionic concentration; (d) The angle $\theta$ distributions $(c=0.1$ and $0.5 \mathrm{~mol} / \mathrm{L})$.

\subsection{Density distribution}

Figure S6 shows the density distributions of water, decane and ions along $z$-direction in cases under $c=0.5 \mathrm{~mol} / \mathrm{L}, E=0.01 \mathrm{~V} / \AA ̊$ condition. We use the following hyperbolic tangent function to fit the density distribution profiles of the water and decane molecules.

$$
\rho_{i}(z)=\frac{\rho_{i, \text { bulk }}}{2}-\frac{\rho_{i, \text { bulk }}}{2} \tanh \left(\frac{2\left(z-z_{0}\right)}{d}\right)
$$


where $\rho_{i}$ is the density, $z_{0}$ is the position of the Gibbs interface, $d$ is the alterable parameter concerning with the interfacial thickness. The interfacial thickness is defined as the distance between the positions where the densities of water or decane respectively reach the $90 \%$ of their bulk densities, which are shown in the baby blue region of Figure S6. It is a common practice to determine the interfacial thickness of the liquid-vapor or liquid-liquid interface.

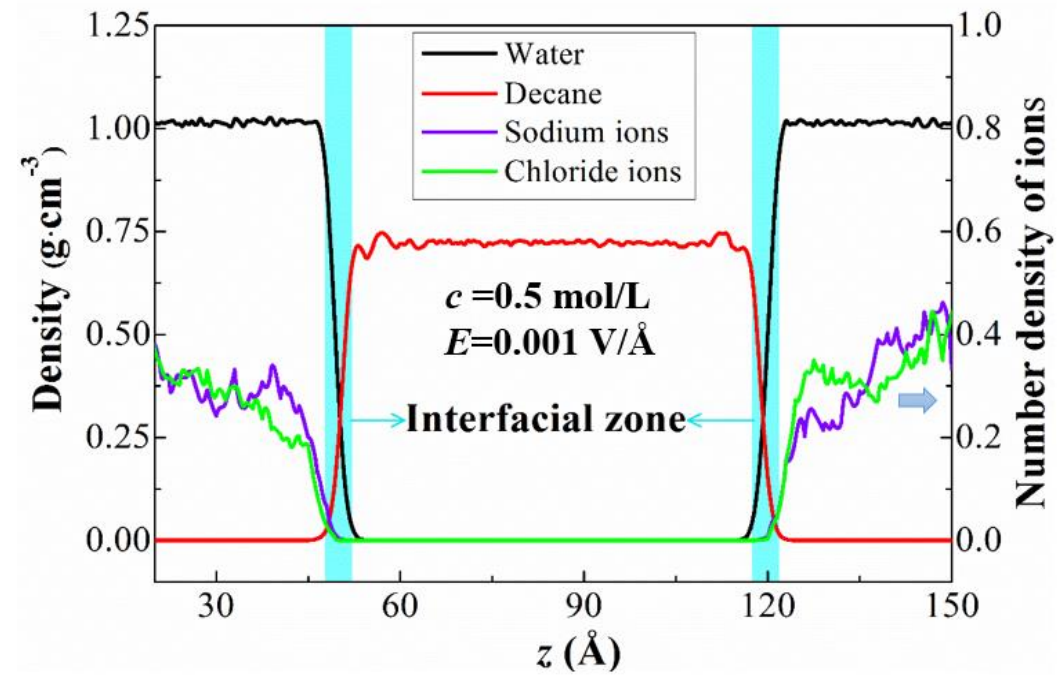

Figure S6. Density distributions of water, decane and ions (number density) along $z$ direction under $c=0.5 \mathrm{~mol} / \mathrm{L}, E=0.001 \mathrm{~V} / \AA ̊$ condition

In the manuscript, we detailedly discuss the density distribution of ions with ionic concentration or electric field intensity increasing. Here, we perform a supplementary analysis on the density distribution of water and decane molecules along $z$-direction under $E=0.001 \mathrm{eV} / \AA$ or $c=0.5 \mathrm{~mol} / \mathrm{L}$ conditions, as shown in Figure S7. With the intensity of electric field increasing, more ions aggregate at the decane-water interfaces and induce a slight decrease of the water and decane densities at the interface, therefore widening the interfacial thickness. With ionic concentration rising, the interfacial thickness remains nearly constant but the interfacial area shifts towards the water phase. 

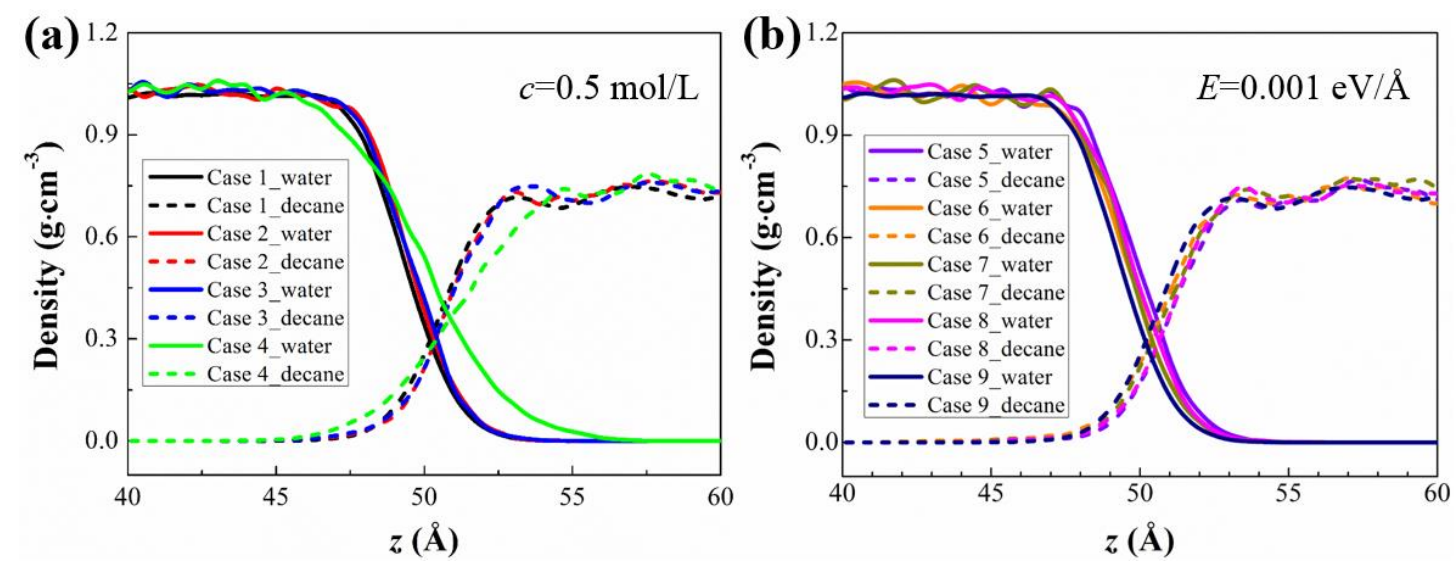

Figure S7. Density distribution of water and decane molecules. (a) Density distribution along $z$-direction $v s$. electric field intensity: Case 1-4: $c=0.5 \mathrm{~mol} / \mathrm{L}, E=0.001,0.005,0.01$ and $0.05 \mathrm{eV} / \AA$, orderly; (b) Density distribution along $z$-direction $v s$. ionic concentration: Case 5-9: $E=0.001 \mathrm{eV} / \AA, c=0.1,0.2,0.3,0.4$ and $0.5 \mathrm{~mol} / \mathrm{L}$, successively.

We also found that the adsorption behavior of ions at the interface is a dynamic process, which is accompanied by the desorption and diffusion of ions at the same time. As shown in Figure S8, ions move back and forth between the water phase and the interface. The adsorption sites of ions at interface are always changing.

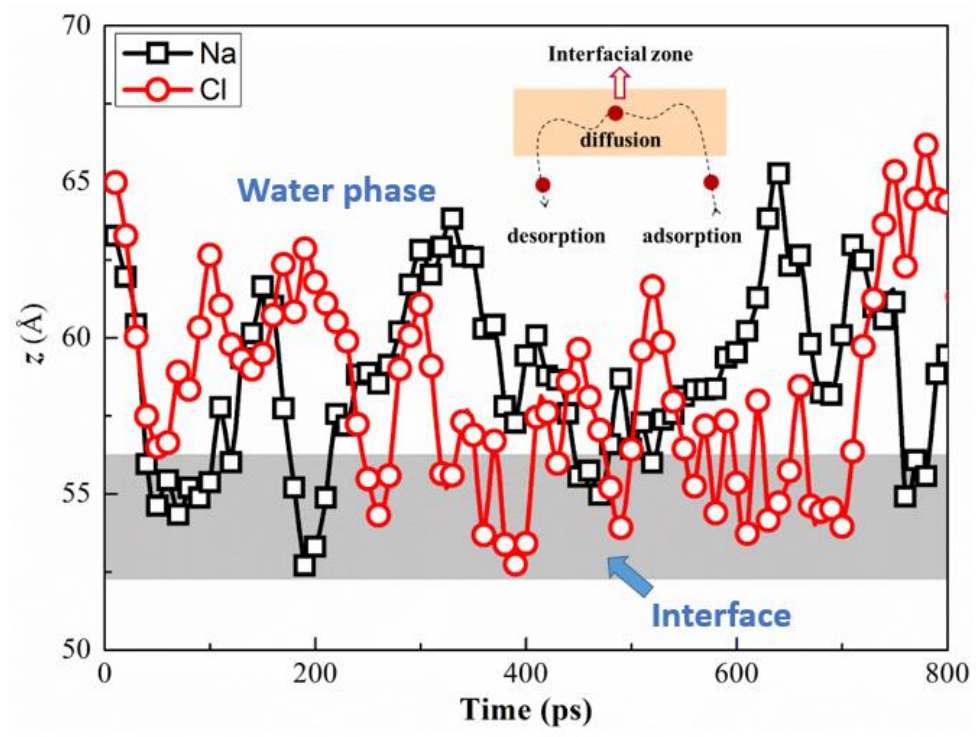

Figure S8. The variation of $z$ coordinate for specific ion versus simulation time. 


\subsection{Interfacial thickness}

Based on the Equation (S7), we can obtain the interfacial thickness of the decanewater interface systems with different ion concentrations or electric field intensities under specific conditions. Figure S9 shows how the interfacial thickness of the decane-water interface change with electric field intensity or ionic concentration increasing. At the ion concentration of $0.5 \mathrm{~mol} / \mathrm{L}$, the interfacial thickness slightly increases as the intensity of electric field rises, which is attributed to the aggregation of more ions at the decane-water interfaces. In addition, with ion concentration rising, the interfacial thickness remains almost unchanged under $E=0.001 \mathrm{~mol} / \mathrm{L}$ condition. To our knowledge, the larger interfacial thickness means the stronger miscibility, therefore inducing a smaller interfacial thickness.
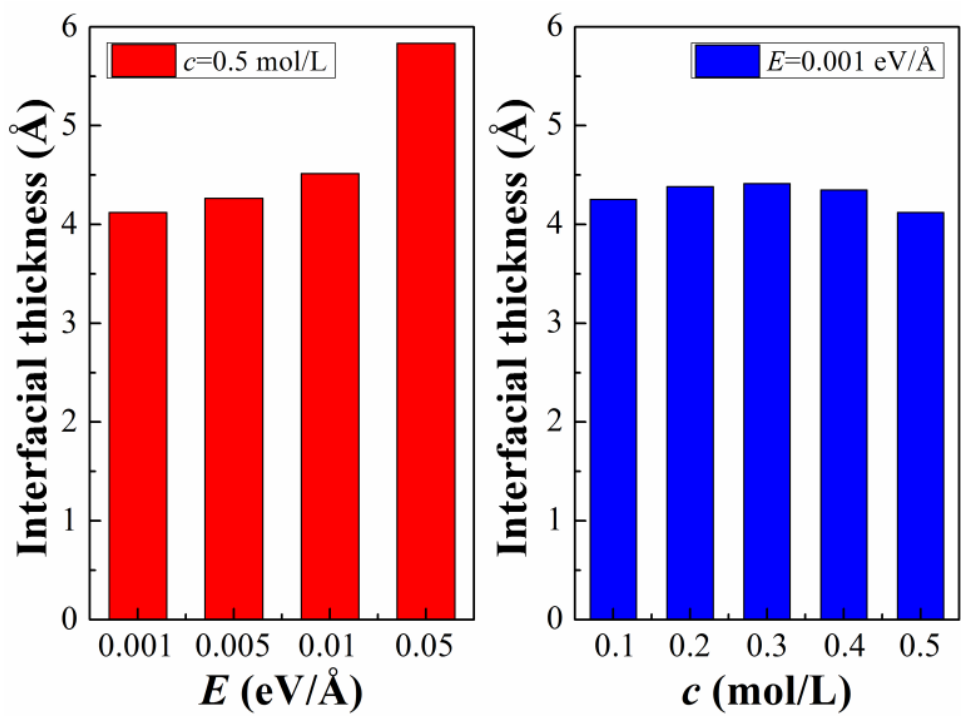

Figure S9. Interfacial thickness. (a) Interfacial thickness $v s$. electric field intensity: $c=0.5$ mol/L; (b) Interfacial thickness $v s$. ionic concentration: $E=0.001 \mathrm{eV} / \AA ̊$. 


\section{References}

1. W. L. Jorgensen, J. Chandrasekhar, J. D. Madura, R. W. Impey and M. L. Klein, J. Chem. Phys., 1983, 79, 926-935.

2. P. Mark and L. Nilsson, J. Phys. Chem. A, 2001, 105, 9954-9960.

3. C. Vega, J. L. F. Abascal, E. Sanz, L. G. Macdowell and C. Mcbride, J. Phys-Condens. Mat., 2005, 17, S3283-S3288.

4. J. Zielkiewicz, J. Chem. Phys., 2005, 123, 1545.

5. C. Feng and P. E. Smith, J. Chem. Phys., 2007, 126, 4873.

6. Q. Ji, J. M. Pellenq and K. J. V. Vliet, Comput. Mater. Sci., 2012, 53, 234-240.

7. D. J. Huggins, J. Chem. Phys., 2012, 136, 851.

8. T. Yagasaki, M. Matsumoto and H. Tanaka, J. Phys. Chem. B, 2018, 122, 7718-7725.

9. B. Wen, C. Sun, B. Bai, E. Y. Gatapova and O. A. Kabov, Phys. Chem. Chem. Phys., 2017, 19, 14606-14614.

10. B. Wen, C. Sun and B. Bai, Phys. Chem. Chem. Phys., 2018, 20, 22796-22804.

11. W. L. Jorgensen, J. Chandrasekhar, J. D. Madura, R. W. Impey and M. L. Klein, J. Chem. Phys., 1983, 79, 926.

12. S. L. Price, A. J. Stone and M. Alderton, Mol. Phys., 1984, 52, 987-1001.

13. S. W. I. Siu, K. Pluhackova and R. A. Böckmann, J. Chem. Theory Comput., 2012, 8, 14591470.

14. A. P. Lyubartsev and A. Laaksonen, J. Phys. Chem., 1996, 100, 16410-16418.

15. S. Nosé, Mol. Phys., 1984, 52, 255-268.

16. W. G. Hoover, Phys. Rev. A-Gen. Phys., 1985, 31, 1695-1697.

17. W. Shinoda, M. Shiga and M. Mikami, Phys. Rev. B, 2004, 69, 134103.

18. G. J. Martyna, D. J. Tobias and M. L. Klein, J. Chem. Phys., 1994, 101, 4177-4189.

19. M. Parrinello and A. Rahman, Phys. Rev. Lett., 1980, 45, 1196-1199.

20. M. E. Tuckerman, J. Alejandre, R. López-Rendón, A. L. Jochim and G. J. Martyna, J. Phys. A- Gen. Phys., 2006, 39, 5629.

21. B. Qiao, J. V. Muntean, M. Olvera de la Cruz and R. J. Ellis, Langmuir, 2017, 33, 6135-6142.

22. J. Tang, Z. Qu, J. Luo, L. He, P. Wang, P. Zhang, X. Tang, Y. Pei, B. Ding, B. Peng and Y. Huang, J. Phys. Chem. B, 2018, 122, 1905-1918. 